\title{
Long-time behavior of an angiogenesis model with flux at the tumor boundary
}

\author{
Tomasz Cieślak and Cristian Morales-Rodrigo
}

\begin{abstract}
This paper deals with a nonlinear system of partial differential equations modeling a simplified tumor-induced angiogenesis taking into account only the interplay between tumor angiogenic factors and endothelial cells. Considered model assumes a nonlinear flux at the tumor boundary and a nonlinear chemotactic response. It is proved that the choice of some key parameters influences the long-time behavior of the system. More precisely, we show the convergence of solutions to different semi-trivial stationary states for different range of parameters.
\end{abstract}

Mathematics Subject Classification. 35K45 - 35K57 - 92C17.

Keywords. Chemotaxis - Asymptotic behavior - Semigroup theory · Nonlinear elliptic eigenvalue problem · Angiogenesis · Nonlinear boundary condition.

\section{Introduction}

Angiogenesis is a physiological process involving the new vessels sprout from a pre-existing vasculature in response to a chemical stimuli. Angiogenesis is an important ingredient of a processes like development, growth and wound healing. However, angiogenesis is also induced by tumoral cells. In this paper, we consider a model of tumor-induced angiogenesis that was proposed in [5]. Actually, in the above-mentioned model, some factors influencing angiogenesis are neglected to keep the model simple but sufficiently interesting from the analytical point of view. We refer the reader to [12] as a source of information about the progress in mathematical modeling and biological knowledge of angiogenesis process. We focus our attention on two key variables: the endothelial cells (ECs), denoted by $u$, and the tumor angiogenic factors (TAF), denoted by $v$. We assume that (ECs) form the blood vessels wall are induced by the (TAF), factors that are generated by the tumor, to migrate chemotactically toward the tumor. We assume that the (ECs) and the (TAF) fill in a bounded and connected domain $\Omega \subset \mathbb{R}^{d}$ with a regular boundary $\partial \Omega$. In particular, neither the existence of extracellular matrix nor the activity of metalloproteinases is considered. But, following [5], nonlinear flux of TAF on the tumor boundary is taken into account. This way we include the fact that ECs are supposed to react chemotactically to the TAF, thus generating the large gradient of TAF on the boundary. This, in turn, is supposed to make the tumor more dangerous. The aim of [5] was to study the interplay between the density of ECs and TAF dependently on a parameter $\mu$ measuring the strength of the flux on the tumor boundary and the nonlinearity $V$ measuring nonlinear response of ECs. In [5], the qualitative features of a model were studied in a local sense. We mean by that the local stability of steady states which were proven to exist in [5]. We complete the studies taken in [5] by analyzing the global stability of steady states. We shall prove the asymptotic convergence of solutions for different values of $\mu$. To be more precise, we consider the case

$$
\partial \Omega=\Gamma_{1} \cup \Gamma_{2},
$$


where $\Gamma_{1} \cap \Gamma_{2}=\emptyset$ and $\Gamma_{i}$ are closed and open sets in the relative topology of $\partial \Omega$. We suppose that $\Gamma_{2}$ is the tumor boundary and $\Gamma_{1}$ is the blood vessel boundary. Our parabolic problem reads.

$$
\begin{cases}u_{t}-\Delta u=-\operatorname{div}(V(u) \nabla v)+\lambda u-u^{2} & \text { in } \Omega \times(0, T), \\ v_{t}-\Delta v=-v-c u v & \text { in } \Omega \times(0, T), \\ \frac{\partial u}{\partial n}=\frac{\partial v}{\partial n}=0 & \text { on } \Gamma_{1} \times(0, T), \\ \frac{\partial u}{\partial n}=0, \quad \frac{\partial v}{\partial n}=\mu \frac{v}{1+v} & \text { on } \Gamma_{2} \times(0, T), \\ u(x, 0)=u_{0}(x), \quad v(x, 0)=v_{0}(x) & \text { in } \Omega,\end{cases}
$$

where $0<T \leq+\infty, \lambda, \mu \in \mathbb{R}, c>0$,

$$
V \in \mathcal{C}^{1}(\mathbb{R}), \quad V>0 \quad \text { in }(0, \infty) \quad \text { with } V(0)=0 ;
$$

and $u_{0}$ and $v_{0}$ are given nonnegative and nontrivial functions. In [5, Theorem 3.1, Theorem 3.8] the existence and uniqueness of global-in-time bounded regular solutions, provided initial data are nonnegative and $V \in L^{\infty}(0,+\infty)$ is shown. Moreover in [5, Section 4], the existence of two semi-trivial steady states $(\lambda, 0), \lambda>0$ and $\left(0, \theta_{\mu}\right)$ is shown provided $\mu>\mu_{1}$ (see also [14]), where $\mu_{1}$ is the principal eigenvalue of the boundary eigenvalue problem

$$
\begin{cases}-\Delta v+v=0 & \text { in } \Omega, \\ \frac{\partial v}{\partial n}=0 & \text { on } \Gamma_{1}, \\ \frac{\partial v}{\partial n}=\mu v & \text { on } \Gamma_{2} .\end{cases}
$$

Furthermore, results concerning the linearized stability around the semi-trivial solutions to (1) are proven in $[5]$.

First models of tumor-induced angiogenesis that we are aware of are considered in [3] (see also [10] for a more elaborated model). A reduced model proposed in [10] is studied in [7]. The local stability of the homogeneous steady states in one-dimensional domains is shown there. In all the mentioned papers, the boundary conditions are either zero Neumann or no flux. In [6], the stationary problem of (1) with linear flux for $v$ is studied. Finally, let us mention [9] where the authors study the local solvability of a system of partial differential equations with a nonlinear boundary condition and a chemotaxis term.

The aim of this paper is to analyze the global stability for positive initial data. In particular, we show global stability for some range of parameters $(\lambda, \mu)$ for which even the local stability is not known.

It should be pointed out that in our investigations, we assume

$$
\|u(t)\|_{\infty}<C, \text { for } t \geq 0 .
$$

In particular, (3) is satisfied when $V$ is bounded (see [5]). However, most of the results of this paper could be extended even to more general forms of $V$, for example, $V(u)=u$ or $V(u)=u^{p}, p>1$, as soon as we know that (3) holds. By the regularity of $V$, we have just to apply the following estimate

$$
V(u) \leq \max _{s \in\left[0,\|u\|_{\infty}\right]} V(s) \leq \max _{s \in[0, C]} V(s)=K<+\infty .
$$

Observe that the parabolic regularity asserts

$$
\|v(t)\|_{\infty}<C
$$

for any $t>0$ once we know (3) and by [5, Theorem 3.1], the solution is global and regular.

\section{Preliminaries}

For the reader's convenience, we collect here some results of interpolation theory and its applications to parabolic problems that will be used throughout the paper. 
a) Let $E_{0}, E_{1}$ be two normed spaces embedded in a common topological Hausdorff space $\mathcal{E}$, we can define the real interpolation function, denoted by

$$
\left(E_{0}, E_{1}\right)_{\theta, p}, \quad 0<\theta<1,1 \leq p \leq+\infty,
$$

(see for instance [13, Def. 22.1]). During the paper, we will use the following property of the real interpolation functor (see [13, Lemma 25.2]):

If $\left(E_{0}, E_{1}\right)_{\theta, p}$ is a Banach space then

$$
\exists C>0 \text { such that }\|x\|_{\left(E_{0}, E_{1}\right)_{\theta, p}} \leq C\|x\|_{E_{0}}^{1-\theta}\|x\|_{E_{1}}^{\theta} \quad \forall x \in E_{0} \cap E_{1} .
$$

In the context of fractional Sobolev spaces, this inequality reads cf. [1, (5.20), (5.21)]

$$
\|x\|_{W^{m, p}} \leq C\|x\|_{W^{k, p}}^{\theta}\|x\|_{p}^{1-\theta}
$$

for $m<k \theta, \theta \in(0,1)$.

b) Let us consider a parabolic problem with a nonhomogeneous boundary condition

$$
\begin{cases}z_{t}+\mathcal{A} z=f(t) & \text { in } \Omega \times(0, T) \\ \mathcal{B} z=g(t) & \text { on } \partial \Omega \times(0, T) \\ z(x, 0)=z_{0}(x), & \text { in } \Omega\end{cases}
$$

where

$$
\mathcal{B} z:=\frac{\partial z}{\partial n}
$$

and

$$
\mathcal{A} z:=-\Delta z+z
$$

We define the space of functions

$$
W_{\mathcal{B}}^{s, p}:= \begin{cases}\left\{z \in W^{s, p}(\Omega): \mathcal{B} z=0\right\} & \text { if } 1+1 / p<s \leq 2, \\ W^{s, p}(\Omega) & \text { if }-1+1 / p<s<1+1 / p, \\ \left(W^{-s, p^{\prime}}(\Omega)\right)^{\prime} & \text { if }-2+1 / p<s \leq-1+1 / p .\end{cases}
$$

It is known that $(\mathcal{A}, \mathcal{B})$, as being in separated divergence form (see [1, pg. 21]), is normally elliptic. We denote by $A_{\alpha-1}$ the $W_{\mathcal{B}}^{2 \alpha-2, p}$-realization of $(\mathcal{A}, \mathcal{B})$ (see [1, pg. 39] for the precise definition). Since $(\mathcal{A}, \mathcal{B})$ is normally elliptic then $A_{\alpha-1}$ generates an analytic semigroup [1, Theorem 8.5]. Moreover, if

$$
(f, g) \in \mathcal{C}\left((0, T) ; W_{\mathcal{B}}^{2 \alpha-2, p}(\Omega) \times W_{\mathcal{B}}^{2 \alpha-1-1 / p, p}(\partial \Omega)\right)
$$

for some $T>0$ and $2 \alpha \in(1 / p, 1+1 / p)$ then for any $t<T$, we rewrite (5) by the generalized variation of constants formula

$$
z(t)=e^{-t A_{\alpha-1}} z_{0}+\int_{0}^{t} e^{-(t-\tau) A_{\alpha-1}}\left(f(\tau)+A_{\alpha-1} \mathcal{B}_{\alpha}^{c} g(\tau)\right) \mathrm{d} \tau,
$$

where $\mathcal{B}_{\alpha}^{c}$ is the continuous extension of $\left(\left.\mathcal{B}\right|_{\operatorname{Ker}(\mathcal{A})}\right)^{-1}$ to $W^{2 \alpha-1-1 / p, p}(\partial \Omega)$. Since $[0,+\infty) \subset \rho\left(-A_{\alpha-1}\right)$ ( $\rho$ is the resolvent set) then by $[1$, Remark $8.6 \mathrm{c}$ )] for $1<p<\infty$, there exists a constant $C \geq 1$ such that

$$
\|z\|_{W_{\mathcal{B}}^{2 \alpha, p}} \leq C\left\|A_{\alpha-1} z\right\|_{W_{\mathcal{B}}^{2 \alpha-2, p}}
$$

c) Let $a, b, c \in L^{\infty}(\Omega)$, the eigenvalue problem

$$
\begin{cases}\frac{-\Delta z+a(x) z=\lambda z}{\partial z}+b(x) z=0 & \text { in } \Omega, \\ \frac{\partial n}{\partial n}+c(x) z=0 & \text { on } \Gamma_{1},\end{cases}
$$


has a unique principal eigenvalue (i.e., an eigenvalue whose associated eigenfunction can be chosen positive in $\Omega$ ) and it will be denoted by

$$
\lambda_{1}(-\Delta+a ; \mathcal{N}+b ; \mathcal{N}+c) .
$$

\section{Convergence to the semi-trivial solution $(\lambda, 0)$}

In the present section, we deal with the convergence to the semi-trivial steady state $(\lambda, 0)$. Throughout this section, we assume (3). A sufficient condition guaranteeing (3) is the boundedness of $V$ (see [5]). We will use the generalized variation of constants formula to estimate $v$, which is stated in the next lemma.

Lemma 3.1. Let $\gamma \in(1,+\infty), \frac{1}{\gamma}<2 \alpha<1+\frac{1}{\gamma}$ and $\beta \in(1,2 \alpha)$. Let $\Sigma$ be a spectral bound of $A_{\alpha-1}$, that is, $\Sigma:=\inf \operatorname{Re} \sigma\left(A_{\alpha-1}\right)$. Then, there exist constants $\delta \in(0, \Sigma)$ and $\theta=\theta(\beta) \in(0,1)$ such that

$$
\left\|e^{-(t-\tau) A_{\alpha-1}} z\right\|_{W^{\beta, \gamma}} \leq C(t-\tau)^{-\theta} e^{-\delta(t-\tau)}\|z\|_{W_{\mathcal{B}}^{2 \alpha-2, \gamma}}
$$

for every $z \in W_{\mathcal{B}}^{2 \alpha, \gamma}$ and every $\tau \in(0, t)$.

Proof. By the choice of $\beta$, we have $W_{\mathcal{B}}^{\beta, \gamma}=W^{\beta, \gamma}(\Omega)$. As a consequence if we apply [1, Theorem 7.2], we get

$$
\left\|e^{-(t-\tau) A_{\alpha-1}} z\right\|_{W^{\beta, \gamma}} \leq C\left\|e^{-(t-\tau) A_{\alpha-1}} z\right\|_{W_{\mathcal{B}}^{2 \alpha, \gamma}}^{\theta} \| e^{-(t-\tau) A_{\alpha-1} z \|_{W_{\mathcal{B}}^{2 \alpha-2, \gamma}}^{1-\theta}}
$$

for some $\theta \in(0,1)$. Next, we apply (6) to the first norm on the right-hand side and [8, Theorem 1.3.4] to deduce

$$
\left\|e^{-(t-\tau) A_{\alpha-1}} z\right\|_{W^{\beta, \gamma}} \leq C(t-\tau)^{-\theta} e^{-\delta(t-\tau) \theta} e^{-\delta(t-\tau)(1-\theta)}\|z\|_{W_{\mathcal{B}}^{2 \alpha-2, \gamma}}
$$

where $\delta \in(0, \Sigma)$.

Lemma 3.2. Let $\gamma \in(1,+\infty), \beta \in(1,1+1 / \gamma), \mu \in\left[0, \mu_{1}\right)$ and $0<\delta<\rho<\alpha(\mu)$ where $\alpha(\mu)$ is defined as

$$
\alpha(\mu):=\lambda_{1}(-\Delta+1 ; \mathcal{N}, \mathcal{N}-\mu) .
$$

Then, there exists $C>0$ such that, for $t>0$, the $v$-solution to (1) satisfies

$$
\begin{aligned}
v(x, t) & \leq C e^{-\rho t} \quad \forall(x, t) \in \bar{\Omega} \times(0,+\infty), \\
\|v(t)\|_{W^{\beta, \gamma}} & \leq C\left(1+t^{-\theta}\right) e^{-\delta t}\left\|v_{0}\right\|_{\gamma},
\end{aligned}
$$

where $\theta=\theta(\beta) \in(0,1)$.

Proof. A solution to the problem

$$
\begin{cases}w_{t}-\Delta w+w=0 & \text { in } \Omega \times\left(0, T_{\max }\right), \\ \frac{\partial w}{\partial n}=0 & \text { on } \Gamma_{1} \times\left(0, T_{\max }\right), \\ \frac{\partial w}{\partial n}=\mu w & \text { on } \Gamma_{2} \times\left(0, T_{\max }\right), \\ w(x, 0)=v_{0}(x) & \text { in } \Omega,\end{cases}
$$

is a supersolution to the $v$-equation of $(1)$; therefore, $v(x, t) \leq w(x, t)$. Since, for sufficiently large $M, \bar{w}=$ $M e^{-\rho t} \varphi_{1}$, with $\varphi_{1}$ a positive eigenfunction associated with $\alpha(\mu)$, is a supersolution to (7), the pointwise estimate in the claim of the lemma follows. For the second one, we pick

$$
\begin{aligned}
f(t) & :=-c u(t) v(t), \\
g(t) & := \begin{cases}0 & \text { on } \Gamma_{1}, \\
\mu \frac{v(t)}{1+v(t)} & \text { on } \Gamma_{2} .\end{cases}
\end{aligned}
$$


Taking the $W^{\beta, \gamma}$-norm in a generalized variation of constants formula for $v$ and using Lemma 3.1, we obtain

$$
\begin{aligned}
\|v(t)\|_{W^{\beta, \gamma}} & \leq\left\|e^{-t A_{\alpha-1}} v_{0}\right\|_{W^{\beta, \gamma}}+\int_{0}^{t}\left\|e^{-(t-\tau) A_{\alpha-1}}\left(f(\tau)+A_{\alpha-1} \mathcal{B}_{\alpha}^{c} g(\tau)\right)\right\|_{W^{\beta, \gamma}} \\
& \leq C\left(e^{-\delta t} t^{-\theta}\left\|v_{0}\right\|_{W_{\mathcal{B}}^{2 \alpha-2, \gamma}}+\int_{0}^{t}(t-\tau)^{-\theta} e^{-\delta(t-\tau)}\left\|f(\tau)+A_{\alpha-1} \mathcal{B}_{\alpha}^{c} g(\tau)\right\|_{W_{\mathcal{B}}^{2 \alpha-2, \gamma}} d \tau\right) .
\end{aligned}
$$

Next, we estimate the last term in the above inequality using the fact that

$$
A_{\alpha-1} \mathcal{B}_{\alpha}^{c} \in \mathcal{L}\left(W^{2 \alpha-1-1 / \gamma, \gamma}(\partial \Omega), W_{\mathcal{B}}^{2 \alpha-2, \gamma}(\Omega)\right)
$$

and the continuous embeddings

$$
L^{\gamma}(\Omega) \hookrightarrow W_{\mathcal{B}}^{2 \alpha-2, \gamma}, \quad L^{\gamma}(\partial \Omega) \hookrightarrow W^{2 \alpha-1-1 / \gamma, \gamma}(\partial \Omega) .
$$

Therefore, we get

$$
\|v(t)\|_{W^{\beta, \gamma}} \leq C e^{-\delta t} t^{-\theta}\left\|v_{0}\right\|_{\gamma}+C e^{-\delta t} \int_{0}^{t} e^{\delta \tau}(t-\tau)^{-\theta}\left(\|f(\tau)\|_{L^{\gamma}(\Omega)}+\|g(\tau)\|_{L^{\gamma}(\partial \Omega)}\right) \mathrm{d} \tau .
$$

Observe that by (3) and the first part of the Lemma, we have

$$
\begin{aligned}
\|f(\tau)\|_{L^{\gamma}(\Omega)} & \leq C\|v\|_{L^{\infty}(\Omega)} \leq C e^{-\rho \tau}, \\
\|g(\tau)\|_{L^{\gamma}(\partial \Omega)} & \leq\|v\|_{L^{\infty}(\partial \Omega)} \leq C e^{-\rho \tau} .
\end{aligned}
$$

In view of the above bounds, (8) yields

$$
\|v(t)\|_{W^{\beta, \gamma}} \leq C e^{-\delta t} t^{-\theta}\left\|v_{0}\right\|_{\gamma}+C e^{-\delta t} \int_{0}^{t} e^{(\delta-\rho) \tau}(t-\tau)^{-\theta} \mathrm{d} \tau .
$$

Next, by the choice of $\delta$ and $\rho, \int_{0}^{\infty} e^{(\delta-\rho) \tau}(t-\tau)^{-\theta} d \tau=C<+\infty$ and the Lemma follows.

Our purpose is to show that $u$ converges to steady states. To this end, we treat separately the cases $\lambda=0, \lambda>0$.

\subsection{Case $\lambda=0$}

Lemma 3.3. Let $\tau>0$ and $y \in C^{1}(\tau,+\infty) \cap L^{1}(\tau,+\infty), y^{\prime} \in L^{1}(\tau,+\infty)$. Then, $\lim _{t \rightarrow+\infty}|y(t)|=0$.

Proof. By the assumptions of the lemma, we observe that for any $k>0$

$$
\lim _{t \rightarrow+\infty} \int_{t}^{t+k}\left(|y(s)|+\left|y^{\prime}(s)\right|\right) \mathrm{d} s=0 .
$$

Let us assume that $\lim _{t \rightarrow+\infty}|y(t)| \neq 0$, then there exists a sequence $\left\{t_{n}\right\}_{n \in \mathbb{N}}, t_{n} \rightarrow+\infty$, such that

$$
\left|y\left(t_{n}\right)\right|>C>0
$$


for all $n \geq n_{0}$. We pick $\theta \in(0, k]$ and see that by $(9)$

$$
|| y\left(t_{n}+\theta\right)|-| y\left(t_{n}\right)|| \leq\left|y\left(t_{n}+\theta\right)-y\left(t_{n}\right)\right| \leq \int_{t_{n}}^{t_{n}+\theta}\left|y^{\prime}(s)\right| \mathrm{d} s \leq \int_{t_{n}}^{t_{n}+k}\left|y^{\prime}(s)\right| \mathrm{d} s<\frac{C}{2}
$$

Therefore, $|y(s)|>C / 2$ for all $s \in\left[t_{n}, t_{n}+k\right], n \geq n_{0}$. The last assertion contradicts the fact that

$$
\lim _{n \rightarrow+\infty} \int_{t_{n}}^{t_{n}+k}|y(s)| \mathrm{d} s=0 .
$$

Next, assume that $(u, v)$ is a global solution to (1).

Lemma 3.4. Let $\lambda=0$ and $t>\tau>0$, then it holds

$$
\mu \int_{\tau}^{t} \int_{\Gamma_{2}} \frac{V(u) v}{1+v}+\int_{\tau}^{t} \int_{\Omega} u^{2}=\int_{\Omega} u(\tau)-\int_{\Omega} u(t) .
$$

Proof. Integrating the $u$-equation of (1) yields

$$
\begin{aligned}
\int_{\Omega} u_{t} & =\int_{\partial \Omega}\left(\frac{\partial u}{\partial n}-V(u) \frac{\partial v}{\partial n}\right)-\int_{\Omega} u^{2} \\
& =-\mu \int_{\Gamma_{2}} \frac{V(u) v}{1+v}-\int_{\Omega} u^{2} .
\end{aligned}
$$

So, integrating the last expression in time between $\tau$ and $t$, we get the result.

Remark 3.5. By Lemma 3.4, we see that for any $t>\tau$

$$
\int_{\tau}^{t} \int_{\Omega} u^{2} \leq\|u(\tau)\|_{1}
$$

Theorem 3.6. Assume that $0 \leq \mu<\mu_{1}$ and $\lambda=0$, then

$$
\lim _{t \rightarrow+\infty}\|u(t)\|_{W^{m, p}}=0,
$$

for any $m<1$ and $p \geq 2$.

Proof. On multiplying the $u$-equation of (1) by $u$ and integrating in space, we obtain

$$
\begin{aligned}
\frac{\mathrm{d}}{2 \mathrm{~d} t} \int_{\Omega} u^{2} & =\int_{\Omega}\left(-|\nabla u|^{2}+V(u) \nabla v \cdot \nabla u-u^{3}\right)-\mu \int_{\Gamma_{2}} \frac{V(u) u v}{1+v} \\
& \leq(\epsilon-1) \int_{\Omega}|\nabla u|^{2}+C(\epsilon) \int_{\Omega}|\nabla v|^{2}-\mu \int_{\Gamma_{2}} \frac{V(u) u v}{1+v}-\int_{\Omega} u^{3} .
\end{aligned}
$$

Therefore, we infer

$$
\frac{\mathrm{d}}{2 \mathrm{~d} t} \int_{\Omega} u^{2}+(1-\epsilon) \int_{\Omega}|\nabla u|^{2} \leq C(\epsilon)\|v\|_{W^{1,2}}^{2}
$$


and after integrating in time, thanks to Lemma 3.2 we arrive at

$$
\int_{\Omega} u(t)^{2}-\int_{\Omega} u(\tau)^{2}+(1-\epsilon) \int_{\tau}^{t} \int_{\Omega}|\nabla u|^{2} \leq C(\epsilon) \int_{\tau}^{t}\left(1+s^{-\theta}\right)^{2} e^{-2 \delta s}\left\|v_{0}\right\|_{2}^{2} .
$$

In particular, we deduce that for $t>\tau$

$$
\int_{\tau}^{t} \int_{\Omega}|\nabla u|^{2} \leq C
$$

By [5, Theorem 3.8], we find a bound $\|u(t)\|_{C(\bar{\Omega})} \leq C$, therefore,

$$
\left|\frac{\mathrm{d}}{2 \mathrm{~d} t} \int_{\Omega} u^{2}\right| \leq C \int_{\Omega}|\nabla u|^{2}+C(\epsilon)\|v\|_{W^{1,2}}^{2}+C \mu \int_{\Gamma_{2}} \frac{V(u) v}{1+v}+C \int_{\Omega} u^{2} .
$$

Thanks to (10), for $t>\tau$

$$
\int_{\tau}^{t}\left|\frac{\mathrm{d}}{2 \mathrm{~d} t} \int_{\Omega} u^{2}\right| \leq C
$$

Finally, Remark 3.5 and (12) together with Lemma 3.3 entail

$$
\lim _{t \rightarrow+\infty}\|u(t)\|_{2}=0
$$

Also thanks to $\|u(t)\|_{C(\bar{\Omega})} \leq C$ for all $t>0$, we obtain

$$
\lim _{t \rightarrow+\infty}\|u(t)\|_{p}=0
$$

for any $p>2$. Next, we recall that by [5, Lemma 3.7] for any $2 \beta \in(k, 1)$, we find a bound on the $X_{\beta}$ norm of $u$, where $X_{\beta}$ is a usual fractional space connected to a semigroup approach to parabolic equations, see [8]. Next, due to the fact that $2 \beta \in(k, 1)$, we infer from the embedding $X_{\beta} \hookrightarrow W^{k, p}$ (see for instance [8, Theorem 1.6.1]) that for all $k<1$ and $p \geq 2$

$$
\|u(t)\|_{W^{k, p}} \leq C
$$

Next, (4) entails

$$
\|u(t)\|_{W^{m, p}} \leq C\|u(t)\|_{W^{k, p}}^{\theta}\|u(t)\|_{p}^{1-\theta}
$$

Therefore, it holds

$$
\lim _{t \rightarrow+\infty}\|u(t)\|_{W^{m, p}} \leq C \lim _{t \rightarrow+\infty}\|u(t)\|_{p}^{1-\theta}=0
$$

Remark 3.7. Let us point out that if we pick $m$ such that $m-d / p>0$ then $W^{m, p}(\Omega)$ is embedded in $\mathcal{C}(\bar{\Omega})$. 


\subsection{Case $\lambda>0$}

Assume that there exists $\delta_{0}$ and $t_{0}$ such that

$$
u(t)>\delta_{0}>0
$$

for $t>t_{0}>0$. Next, we examine the long-time behavior for $u$ under the hypothesis (14). In the sequel, we shall give sufficient conditions on $V(u)$ implying (14).

Theorem 3.8. Let $0 \leq \mu<\mu_{1}$ and assume (14) is satisfied, then there exists $\theta>0$ such that

$$
\|u(t)-\lambda\|_{W^{m, p}} \leq C e^{-\theta t}
$$

for all $t \geq t_{0}$ and any $m<1, p \geq 2$.

Proof. On multiplying the $u$-equation by $u-\lambda$, we have

$$
\begin{aligned}
\frac{\mathrm{d}}{2 \mathrm{~d} t} \int_{\Omega}(u-\lambda)^{2}= & -\int_{\Omega}|\nabla u|^{2}+\int_{\Omega} V(u) \nabla v \cdot \nabla u-\mu \int_{\Gamma_{2}} \frac{v V(u)}{1+v}(u-\lambda)-\int_{\Omega} u(u-\lambda)^{2} \\
\leq & -\frac{1}{2} \int_{\Omega}|\nabla u|^{2}+\frac{\|V\|_{\infty}^{2}}{2} \int_{\Omega}|\nabla v|^{2} \\
& +\mu\|V(u)(u-\lambda)\|_{2, \Gamma_{2}}\left(\int_{\Gamma_{2}} \frac{v^{2}}{(1+v)^{2}}\right)^{1 / 2}-\int_{\Omega} u(u-\lambda)^{2} .
\end{aligned}
$$

Having in mind that $(1+v)^{2} \geq 1$, the hypothesis (14) and the Sobolev trace embedding

$$
W^{1,2}(\Omega) \hookrightarrow L^{2}(\partial \Omega)
$$

we get

$$
\frac{\mathrm{d}}{\mathrm{d} t} \int_{\Omega}(u-\lambda)^{2}+2 \delta_{0} \int_{\Omega}(u-\lambda)^{2} \leq C\|v\|_{W^{1,2}}^{2}+\mu C\|v\|_{W^{1,2}} .
$$

By Lemma 3.2, we can deduce

$$
\|u(t)-\lambda\|_{2}^{2} \leq C e^{-\theta_{1} t}
$$

for $0<\theta_{1}<\min \left\{2 \delta_{0}, \beta\right\}$. At this point, we can argue exactly as in the end of the proof of Theorem 3.6; namely by the bound on $u$ in $L^{\infty}$, we infer the bound on the $L^{p}$ norm of $u, p>2$. Next, we use the estimate of $u$ in $W^{k, p}, k<1, p \geq 2$, coming from [5, Lemma 3.7], in order to conclude (15).

In the rest of this section, we give sufficient conditions on $V$ implying (14). Actually, only the behavior of $V$ around zero matters. Roughly speaking, we require a superlinear growth of $V$ in the neighborhood of zero. From now on, we assume that there exist $C, \delta>0, k_{0}>1+d / 2, j>d / 2$ such that

$$
0<V(s)<C s^{k_{0}},\left|V^{\prime}(s)\right| \leq C s^{j}
$$

for all $s \in(0, \delta)$.

Remark 3.9. The condition (18) is satisfied, for example, for functions

$$
V(u)=\frac{u^{\alpha}}{1+u^{\alpha}}
$$

with $\alpha>1+d / 2$. 
Next, we introduce some notation that will be used in the proof of (14). Moreover, we formulate a lemma which we need in the main part of the proof of $(14)$. Let $f(\delta), g(\delta)$ be defined in a following way:

$$
\begin{aligned}
& f(\delta):=\sup _{s \in(0, \delta)} V^{2}(s), \\
& g(\delta):=\sup _{s \in(0, \delta)}\left(2(s-\delta)^{2} V^{\prime}(s)^{2}+2 V^{2}(s)\right) .
\end{aligned}
$$

Lemma 3.10. Assume that (18) holds. Moreover, for some $D, \mu>0, \eta>1, \tilde{\epsilon}$ and $C(\tilde{\epsilon})$ are given by

$$
\tilde{\epsilon}=\frac{\delta^{2 \eta}}{2 \mu D}, \quad C(\tilde{\epsilon})=\frac{\mu D}{2 \delta^{2 \eta}}
$$

Then, if $\delta>0$ is small enough, the following conditions are satisfied simultaneously

$$
C(\widetilde{\epsilon}) \frac{V^{2}(s)}{s} \delta \leq \lambda-\delta
$$

for $s \in(0, \delta)$,

$$
C(\widetilde{\epsilon}) g(\delta)<1 / 2
$$

and

$$
f(\delta) D \leq \frac{\delta^{2 \eta}}{2}
$$

Proof. Thanks to (18), we have

$$
f(\delta) D=\sup _{s \in(0, \delta)} V^{2}(s) D \leq C \delta^{2 k_{0}} D .
$$

Hence, for $\eta<k_{0}$ and $\delta$ sufficiently small, (21) is satisfied. Next, owing to (18), we observe that

$$
C(\widetilde{\epsilon}) \frac{V^{2}(s)}{s} \delta \leq C(\widetilde{\epsilon}) \delta^{2 k_{0}}
$$

Thus, (19) can be assured for $\eta<k_{0}$ and $\delta$ small enough. Moreover, it is straightforward to see that (20) is also satisfied for $1<\eta<\min \left\{k_{0}, 1+j\right\}$.

Lemma 3.11. Assume that $0 \leq \mu<\mu_{1}$ and that (18) is satisfied then (14) holds.

Proof. Let $\delta>0$ be a fixed constant defined in (18). Given a function $f$, we define the negative part of $f$ as a nonpositive function as follows

$$
f_{-}:=\min \{f, 0\} .
$$


Our purpose is to show that $\left\|(u-\delta)_{-}(t)\right\|_{\infty} \leq \delta / 2$ for every $t>t_{0}$ which implies (14). In order to obtain the previous estimate, we multiply the $u$-equation by $(u-\delta)$ - and we integrate in space to obtain

$$
\begin{aligned}
\frac{\mathrm{d}}{2 \mathrm{~d} t} \int_{\Omega}(u-\delta)_{-}^{2}= & -\int_{\Omega}(\nabla u-V(u) \nabla v) \cdot \nabla(u-\delta)_{-} \\
& +\int_{\partial \Omega}\left(\frac{\partial u}{\partial n}-V(u) \frac{\partial v}{\partial n}\right)(u-\delta)_{-}+\int_{\Omega} u(\lambda-u)(u-\delta)_{-} \\
= & -\int_{\Omega}\left|\nabla(u-\delta)_{-}\right|^{2}+\int_{\Omega} V(u) \nabla v \cdot \nabla(u-\delta)_{-} \\
& -\int_{\Gamma_{2}} V(u) \mu \frac{v}{1+v}(u-\delta)_{-}+\int_{\Omega} u(\lambda-u)(u-\delta)_{-} \\
= & -\int_{\Omega}\left|\nabla(u-\delta)_{-}\right|^{2}+\int_{\Omega_{\delta}} V(u) \nabla v \cdot \nabla(u-\delta)_{-} \\
& -\mu \int_{\Gamma_{\delta}} \frac{v}{1+v} V(u)(u-\delta)_{-}+\int_{\Omega} u(\lambda-u)(u-\delta)_{-},
\end{aligned}
$$

where

$$
\Omega_{\delta}:=\{x \in \Omega: u(x)<\delta\}, \quad \Gamma_{\delta}:=\left\{x \in \Gamma_{2}: u(x)<\delta\right\} .
$$

Consequently,

$$
\begin{aligned}
\frac{\mathrm{d}}{2 \mathrm{~d} t} \int_{\Omega}(u-\delta)_{-}^{2} \leq & (\epsilon-1) \int_{\Omega}\left|\nabla(u-\delta)_{-}\right|^{2}+C(\epsilon) \int_{\Omega_{\delta}} V^{2}(u)|\nabla v|^{2} \\
& -\mu \int_{\Gamma_{\delta}} \frac{v}{1+v} V(u)(u-\delta)_{-}+\int_{\Omega} u(\lambda-u)(u-\delta)_{-} \\
\leq & (\epsilon-1) \int_{\Omega}\left|\nabla(u-\delta)_{-}\right|^{2}+C(\epsilon) \sup _{s \in(0, \delta)} V^{2}(s) \int_{\Omega}|\nabla v|^{2} \\
& -\mu \int_{\Gamma_{\delta}} \frac{v}{1+v} V(u)(u-\delta)_{-}+\int_{\Omega} u(\lambda-u)(u-\delta)_{-} .
\end{aligned}
$$

Previous inequality can be rewritten in terms of $f(\delta)$ defined before Lemma 3.10 as

$$
\begin{aligned}
\frac{\mathrm{d}}{2 \mathrm{~d} t} \int_{\Omega}(u-\delta)_{-}^{2} \leq & (\epsilon-1) \int_{\Omega}\left|\nabla(u-\delta)_{-}\right|^{2}+C(\epsilon) f(\delta) \int_{\Omega}|\nabla v|^{2}+\mu \widetilde{\epsilon} \int_{\Gamma_{2}} \frac{v^{2}}{(1+v)^{2}} \\
& +\mu C(\widetilde{\epsilon}) \int_{\Gamma_{2}} V(u)^{2}(u-\delta)_{-}^{2}+\int_{\Omega} u(\lambda-u)(u-\delta)_{-} .
\end{aligned}
$$


Thanks to the Sobolev trace embedding $W^{1,2}(\Omega) \hookrightarrow L^{2}(\partial \Omega)$ and having in mind that $(v+1)^{2} \geq 1$, we arrive at

$$
\begin{aligned}
& \int_{\Gamma_{2}} V(u)^{2}(u-\delta)_{-}^{2} \leq C\left(\int_{\Omega} V^{2}(u)(u-\delta)_{-}^{2}+\int_{\Omega}\left(2(u-\delta)_{-}^{2} V^{\prime}(u)^{2}+2 V^{2}(u)\right)\left|\nabla(u-\delta)_{-}\right|^{2}\right), \\
& \mu \widetilde{\epsilon} \int_{\Gamma_{2}} \frac{v^{2}}{(1+v)^{2}} \leq C \mu \widetilde{\epsilon}\|v\|_{W^{1,2}}^{2} .
\end{aligned}
$$

Therefore, we obtain

$$
\begin{aligned}
\frac{\mathrm{d}}{2 \mathrm{~d} t} \int_{\Omega}(u-\delta)_{-}^{2} \leq & (\epsilon-1) \int_{\Omega}\left|\nabla(u-\delta)_{-}\right|^{2}+C(\epsilon) f(\delta) \int_{\Omega}|\nabla v|^{2}+C \widetilde{\epsilon}\|v\|_{W^{1,2}}^{2} \\
& +C(\widetilde{\epsilon})\left(\int_{\Omega} V^{2}(u)(u-\delta)_{-}^{2}+\int_{\Omega}\left(2(u-\delta)_{-}^{2} V^{\prime}(u)^{2}+2 V^{2}(u)\right)\left|\nabla(u-\delta)_{-}\right|^{2}\right) \\
& +\int_{\Omega} u(\lambda-u)(u-\delta)_{-} .
\end{aligned}
$$

In view of the nonnegativity of $u$, we have

$$
-\delta<(u-\delta)_{-} .
$$

Owing to (23), from $(22)$, we see that $(g(\delta)$ was defined before Lemma 3.10)

$$
\begin{aligned}
\frac{\mathrm{d}}{2 \mathrm{~d} t} \int_{\Omega}(u-\delta)_{-}^{2} \leq & (\epsilon+C(\widetilde{\epsilon}) g(\delta)-1) \int_{\Omega}\left|\nabla(u-\delta)_{-}\right|^{2} \\
& +(C(\epsilon) f(\delta)+\mu \widetilde{\epsilon})\|v\|_{W^{1,2}}^{2}+\int_{\Omega} u(u-\delta)_{-}\left(\lambda-u-C(\widetilde{\epsilon}) \frac{V^{2}(u)}{u} \delta\right) .
\end{aligned}
$$

Due to the nonpositivity of $(u-\delta)_{-}$and $(20)$, we have

$$
\int_{\Omega} u(u-\delta)_{-}\left(\lambda-u-C(\widetilde{\epsilon}) \frac{V^{2}(u)}{u} \delta\right)<0 .
$$

By the Hopf lemma and zero Neumann data on the boundary for $u$, we see that there exists $\delta_{1}$ such that $u\left(t_{0}\right)>\delta_{1}$. Hence, choosing $\delta<\delta_{1}$ and using (19), (25) and Lemma 3.2, we infer from (24)

$$
\left\|(u-\delta)_{-}(t)\right\|_{2}^{2} \leq(2 C(\epsilon) f(\delta)+2 \mu \widetilde{\epsilon}) C(\beta),
$$

for $t>t_{0}>0$. We shall show that

$$
\left\|(u-\delta)_{-}(t)\right\|_{2}^{2} \leq \delta^{2 \eta},
$$

for some $\eta>1$. To this end notice that choosing $\epsilon=C(\epsilon)=1 / 2$, we are in a position to apply Lemma 3.10 with $D=C(\beta)$. As a consequence, for $\tilde{\epsilon}$ as it is chosen in Lemma 3.10, (19), (20), (21) and

$$
2 \mu \tilde{\epsilon} C(\beta) \leq \frac{\delta^{2 \eta}}{2}
$$

are satisfied simultaneously. Hence, (26) is shown.

Next, we use interpolation between $L^{p}$ spaces, (26) and (23) to obtain

$$
\begin{aligned}
\left\|(u-\delta)_{-}\right\|_{2 / \theta_{1}} & \leq\left\|(u-\delta)_{-}\right\|_{2}^{\theta_{1}}\left\|(u-\delta)_{-}\right\|_{\infty}^{1-\theta_{1}} \\
& \leq \delta^{\alpha \theta_{1}} \delta^{1-\theta_{1}}=\delta^{1+(\alpha-1) \theta_{1}} .
\end{aligned}
$$


Applying (4), we infer

$$
\left\|(u-\delta)_{-}\right\|_{W^{\theta, 2 / \theta_{1}}} \leq C\left\|(u-\delta)_{-}\right\|_{W^{1,2 / \theta_{1}}}^{\theta}\left\|(u-\delta)_{-}\right\|_{2 / \theta_{1}}^{1-\theta} \leq C_{1}\left\|(u-\delta)_{-}\right\|_{2 / \theta_{1}}^{1-\theta},
$$

the last inequality being a consequence of the uniform bound of $L^{\infty}$ norm, see [5, Theorem 3.8], and [1, Theorem 15.5]. Picking up $\theta_{1}$ such that

$$
\theta-\frac{d \theta_{1}}{2}>0
$$

we make sure that $W^{\theta, 2 / \theta_{1}}(\Omega) \hookrightarrow L^{\infty}(\Omega)$. Consequently,

$$
\left\|(u-\delta)_{-}\right\|_{\infty} \leq C_{2} \delta^{(1-\theta)\left(1+(\alpha-1) \theta_{1}\right)} .
$$

Next, we notice that choosing $\alpha>1+\frac{d}{2}$, we make sure that

$$
1<\left(1-\frac{d \theta_{1}}{2}\right)\left(1+(\alpha-1) \theta_{1}\right) .
$$

Hence, choosing $\theta$ close enough to $\frac{d \theta_{1}}{2}$, we see that $(1-\theta)\left(1+(\alpha-1) \theta_{1}\right)>1$ and upon taking $\delta$ small enough, we obtain

$$
\left\|(u-\delta)_{-}(t)\right\|_{\infty} \leq \frac{\delta}{2}
$$

for $t \geq t_{0}>0$. The Lemma is proved.

\section{Convergence to the semi-trivial solution $\left(0, \theta_{\mu}\right)$}

Throughout this Section, additionally to the boundedness of $u$, we assume that there exist constants $0<c_{m}<C_{M}$ and $\alpha \geq 1$ such that

$$
c_{m} s^{\alpha} \leq V(s) \leq C_{M} s^{\alpha} \text { for all } s \in\left[0,\|u\|_{\infty}\right] .
$$

Remark 4.1. Let us observe that when $V^{\prime}(0) \neq 0$ and (2) holds, then (28) is true for $\alpha=1$. Moreover, if $V \in \mathcal{C}^{k}$ for $k \geq 1$ with $V^{k}(0) \neq 0$ and $V^{j}(0)=0$ for $j<k$, then (28) holds true for $\alpha=k$.

In the following Theorem, we eliminate the restriction on $\mu$ of Theorem 3.6. However, we require the additional condition (28) on $V$.

Theorem 4.2. Let $\lambda=0$ and assume (28), then

$$
\lim _{t \rightarrow+\infty}\|u(t)\|_{W^{m, p}}=0
$$

for any $m<1$ and $p \geq 2$.

Proof. On the one hand, we multiply the $u$-equation of (1) by $u$ and we integrate in the space variable to obtain

$$
\begin{aligned}
\frac{\mathrm{d}}{2 \mathrm{~d} t} \int_{\Omega} u^{2} & =\int_{\Omega}\left(-|\nabla u|^{2}+V(u) \nabla v \cdot \nabla u-u^{3}\right)-\mu \int_{\Gamma_{2}} \frac{V(u) u v}{1+v} \\
& =\int_{\Omega}\left(-|\nabla u|^{2}+\nabla v \cdot \nabla \varphi(u)-u^{3}\right)-\mu \int_{\Gamma_{2}} \frac{V(u) u v}{1+v}
\end{aligned}
$$

with

$$
\varphi(u)=\int_{0}^{u} V(s) \mathrm{d} s .
$$


On the other hand, we multiply the $v$-equation of (1) by $\varphi(u)$. Integrating in space, we obtain

$$
\int_{\Omega} \nabla v \cdot \nabla \varphi(u)=-\int_{\Omega} \varphi(u) v_{t}+\mu \int_{\Gamma_{2}} \frac{v \varphi(u)}{1+v}-\int_{\Omega} v \varphi(u)-\int_{\Omega} \operatorname{cuv} \varphi(u) .
$$

Inserting the above equality into (29), we have

$$
\frac{\mathrm{d}}{2 \mathrm{~d} t} \int_{\Omega} u^{2}=\int_{\Omega}\left(-|\nabla u|^{2}+\varphi(u)\left(-v_{t}-v-c u v\right)-u^{3}\right)+\mu \int_{\Gamma_{2}} \frac{v}{1+v}(\varphi(u)-V(u) u) .
$$

Next, we estimate $v_{t}$. Multiplying the $v$-equation by $v_{t}$ and integrating over $\Omega$, we see that

$$
\frac{1}{2} \int_{\Omega} v_{t}^{2}+\frac{\mathrm{d}}{2 \mathrm{~d} t} \int_{\Omega}|\nabla v|^{2}+\frac{\mathrm{d}}{2 \mathrm{~d} t} \int_{\Omega} v^{2}-\frac{\mu \mathrm{d}}{\mathrm{d} t} \int_{\Gamma_{2}} \theta(v)=-\int_{\Omega} c u v v_{t},
$$

where

$$
\theta(v):=\int_{0}^{v} \frac{s}{1+s} \mathrm{~d} s
$$

Therefore, by the uniform bound of $v$ in $\mathcal{C}(\bar{\Omega})$, we deduce

$$
\frac{1}{4} \int_{\Omega} v_{t}^{2}+\frac{\mathrm{d}}{2 \mathrm{~d} t} \int_{\Omega}|\nabla v|^{2}+\frac{\mathrm{d}}{2 \mathrm{~d} t} \int_{\Omega} v^{2}-\frac{\mu \mathrm{d}}{\mathrm{d} t} \int_{\Gamma_{2}} \theta(v) \leq M \int_{\Omega} u^{2} .
$$

After integrating over the interval $(\tau, t)$, we find, by Lemma 3.4, that for $t \geq \tau$

$$
\int_{\tau}^{t} \int_{\Omega} v_{t}^{2} \leq C .
$$

Next, by (28), we obtain from (30) that

$$
\begin{aligned}
\frac{\mathrm{d}}{2 \mathrm{~d} t} \int_{\Omega} u^{2} & \leq-\int_{\Omega}|\nabla u|^{2}+\int_{\Omega} \varphi(u)^{2}+\int_{\Omega} v_{t}^{2}+\mu \int_{\Gamma_{2}} \frac{v C_{u} u^{\alpha+1}}{(1+v)(\alpha+1)} \\
& \leq-\int_{\Omega}|\nabla u|^{2}+\max _{s \in\left[0, C_{u}\right]} V^{2}(s) \int_{\Omega} u^{2}+\int_{\Omega} v_{t}^{2}+\frac{\mu C_{u}^{2}}{\alpha+1} \int_{\Gamma_{2}} \frac{v u^{\alpha}}{1+v} .
\end{aligned}
$$

By Lemma 3.4 and (28), we get

$$
\int_{\tau}^{t} \int_{\Gamma_{2}} \frac{v u^{\alpha}}{1+v} \leq C
$$

for $t \geq \tau$. According to (33) and (31), we find upon integration of (32) over the time interval $(\tau, t)$ that for $t \geq \tau$

$$
\int_{\tau}^{t} \int_{\Omega}|\nabla u|^{2} \leq C .
$$

From the last estimate, a similar argument to the one used previously yields

$$
\int_{\tau}^{t}\left|\frac{\mathrm{d}}{\mathrm{d} t} \int_{\Omega} u^{2}\right| \leq C
$$


for $t \geq \tau$. Thus, by Lemma 3.3

$$
\lim _{t \rightarrow+\infty}\|u(t)\|_{2}=0 .
$$

Finally, we can infer the result arguing as in the end of the proof of Theorem 3.6.

Next, we prove a lemma which we will use in the proof of Theorem 4.4. As a by-product of the following lemma, we learn a qualitative information that $v$ is bounded away from 0 for times large enough. We shall obtain a lower bound on $v$ by considering a subsolution to an elliptic problem which is also a subsolution to a second equation in (1).

Lemma 4.3. Let $\lambda=0$ and $\mu>\mu_{1}$. If the condition (28) is satisfied then there exist constants $c_{1}, t_{0}>0$ such that for $t \geq t_{0}$

$$
v(t)>c_{1} .
$$

Proof. Let $k \in\left(\mu_{1}, \mu\right)$. Since $\lambda_{1}(-\Delta+1 ; \mathcal{N} ; \mathcal{N}+b(x))$ is increasing with respect to $b$ (see [4, Proposition $3.3])$, we have

$$
\lambda_{1}(-\Delta+1 ; \mathcal{N} ; \mathcal{N}-\mu)<\lambda_{1}(-\Delta+1 ; \mathcal{N} ; \mathcal{N}-k)<\lambda_{1}\left(-\Delta+1 ; \mathcal{N} ; \mathcal{N}-\mu_{1}\right)=0 .
$$

Therefore, there exists $\epsilon_{0}>0$ such that

$$
\lambda_{1}(-\Delta+1 ; \mathcal{N} ; \mathcal{N}-k)=-c \epsilon_{0} \text { i.e., } \lambda_{1}\left(-\Delta+1+c \epsilon_{0} ; \mathcal{N} ; \mathcal{N}-k\right)=0 .
$$

Let $\varphi_{1}$ be the positive eigenfunction with $\left\|\varphi_{1}\right\|_{\infty}=1$ associated with the above eigenvalue, that is, $\varphi_{1}$ satisfies

$$
\begin{cases}-\Delta \varphi_{1}+\left(1+\epsilon_{0} c\right) \varphi_{1}=0 & \text { in } \Omega, \\ \frac{\partial \varphi_{1}}{\partial n}=0 & \text { on } \Gamma_{1}, \\ \frac{\partial \varphi_{1}}{\partial n}=k \varphi_{1} & \text { on } \Gamma_{2} .\end{cases}
$$

By Theorem 4.2, there exists $t_{0}>0$ such that $0 \leq u(t)<\epsilon_{0}$ for all $t \geq t_{0}>0$. We claim that there exists $\delta>0$ such that $\underline{w}=\delta \varphi_{1}$ is a subsolution to

$$
\begin{cases}w_{t}-\Delta w+(1+c u) w=0 & \text { in } \Omega \times\left(t_{0},+\infty\right), \\ \frac{\partial w}{\partial n}=0 & \text { on } \Gamma_{1} \times\left(t_{0},+\infty\right), \\ \frac{\partial w}{\partial n}=\mu \frac{w}{1+w} & \text { on } \Gamma_{2} \times\left(t_{0},+\infty\right) . \\ w\left(x, t_{0}\right)=v\left(x, t_{0}\right) & \text { in } \Omega .\end{cases}
$$

Therefore, $v(x, t) \geq \delta \varphi_{1} \geq c_{1}$. It remains to prove the claim. By the strong maximum principle, $v\left(x, t_{0}\right)>$ $c>0$. Thus, there exists $\delta>0$ such that $\delta \varphi_{1}<v\left(x, t_{0}\right)$. Moreover, choosing $\delta>0$ such that $k(1+\delta)<\mu$, we make sure that

$$
\frac{\partial \underline{w}}{\partial n} \leq \mu \frac{\underline{w}}{1+\underline{w}}
$$

on $\Gamma_{2} \times\left(t_{0},+\infty\right)$. Hence, the claim is shown, and the lemma follows.

Now, we are in a position to prove the main result of this section. To this end, we make use of the theorem by Amann and López-Gómez, see [2], stating the equivalence between positivity of principal eigenvalue and existence of strictly positive supersolution of some elliptic problems (the previous version of this theorem for the Dirichlet problem was shown in [11]).

Theorem 4.4. Let $\lambda=0, \mu>\mu_{1}$ and assume (28), then

$$
\lim _{t \rightarrow+\infty}\left\|v(t)-\theta_{\mu}\right\|_{2}=0 \text {. }
$$


Proof. Let $z(t)=v(t)-\theta_{\mu}$. Then, $z$ solves the following parabolic problem

$$
\begin{cases}z_{t}=\Delta z-z-c u v & \text { in } \Omega \times(0, T), \\ \frac{\partial v}{\partial n}=0 & \text { on } \Gamma_{1} \times(0, T), \\ \frac{\partial z}{\partial n}=\mu \frac{z}{(1+v)\left(1+\theta_{\mu}\right)} & \text { on } \Gamma_{2} \times(0, T), \\ z(x, 0)=v_{0}(x)-\theta_{\mu} & \text { in } \Omega .\end{cases}
$$

We multiply (35) by $z$ to obtain

$$
\frac{\mathrm{d}}{2 \mathrm{~d} t} \int_{\Omega} z^{2}=-\int_{\Omega}|\nabla z|^{2}+\mu \int_{\Gamma_{2}} \frac{z^{2}}{(1+v)\left(1+\theta_{\mu}\right)}-\int_{\Omega} z^{2}-\int_{\Omega} \text { cuvz. }
$$

In order to estimate the right-hand side of (36) for $t \geq t_{0}$, we pick $\gamma>1$ such that

$$
\frac{\gamma}{1+c_{1}}<1
$$

where $c_{1}$ is given in (34). For each $t \geq t_{0}$, we consider the eigenvalue problem

$$
\begin{cases}-\Delta w+w=\lambda w & \text { in } \Omega, \\ \frac{\partial w}{\partial n}=0 & \text { on } \Gamma_{1}, \\ \frac{\partial w}{\partial n}=\frac{\mu \gamma w}{(1+v(t))\left(1+\theta_{\mu}\right)} & \text { on } \Gamma_{2} .\end{cases}
$$

Next, we see that $\theta_{\mu}$ is a strict supersolution of

$$
\begin{cases}-\Delta w+w=0 & \text { in } \Omega, \\ \frac{\partial w}{\partial n}=0 & \text { on } \Gamma_{1}, \\ \frac{\partial w}{\partial n}=\frac{\mu \gamma w}{(1+v(t))\left(1+\theta_{\mu}\right)} & \text { on } \Gamma_{2} .\end{cases}
$$

Indeed,

$$
\begin{aligned}
-\Delta \theta_{\mu}+\theta_{\mu} & =0 \quad \text { in } \Omega, \\
\frac{\partial \theta_{\mu}}{\partial n} & =0 \quad \text { on } \Gamma_{1},
\end{aligned}
$$

Finally, by the choice of $\gamma$ (see (37)) and Lemma 4.3, we have

$$
\frac{\partial \theta_{\mu}}{\partial n}=\mu \frac{\theta_{\mu}}{1+\theta_{\mu}}>\frac{\mu \gamma \theta_{\mu}}{(1+v(t))\left(1+\theta_{\mu}\right)} \quad \text { on } \Gamma_{2} .
$$

Therefore, by [2, Theorem 2.4] we get $\lambda_{1}>0$, the principal eigenvalue of (38). Next, the variational characterization of the principal eigenvalue entails

$$
\lambda_{1}=\inf _{\varphi \in H^{1}(\Omega)} \frac{\int_{\Omega}|\nabla \varphi|^{2}+\int_{\Omega} \varphi^{2}-\mu \gamma \int_{\Gamma_{2}} \frac{\varphi^{2}}{(1+v(t))\left(1+\theta_{\mu}\right)}}{\int_{\Omega} \varphi^{2}} .
$$

Thus, for all $\varphi \in H^{1}(\Omega)$, we have

$$
\lambda_{1} \gamma^{-1} \int_{\Omega} \varphi^{2} \leq \gamma^{-1} \int_{\Omega}|\nabla \varphi|^{2}+\gamma^{-1} \int_{\Omega} \varphi^{2}-\mu \int_{\Omega} \frac{\varphi^{2}}{(1+v(t))\left(1+\theta_{\mu}\right)} .
$$

In particular, we can apply it in (36) to obtain the following inequality

$$
\frac{\mathrm{d}}{2 \mathrm{~d} t} \int_{\Omega} z^{2}+\left(1-\gamma^{-1}\right) \int_{\Omega}|\nabla z|^{2}+\left(1-\gamma^{-1}+\lambda_{1} \gamma^{-1}\right) \int_{\Omega} z^{2} \leq \int_{\Omega} \text { cuvz. }
$$


Therefore, there exists $M>0$ such that

$$
\frac{\mathrm{d}}{2 \mathrm{~d} t} \int_{\Omega} z^{2}+\left(1-\gamma^{-1}\right)\left(\int_{\Omega}|\nabla z|^{2}+\int_{\Omega} z^{2}\right) \leq M \int_{\Omega} u^{2} .
$$

Integrating the above estimate on the time interval $(\tau, t)$, we obtain for $t \geq \tau$,

$$
\int_{\tau}^{t} \int_{\Omega}|\nabla z|^{2}+\int_{\Omega} z^{2} \leq C .
$$

In view of (39), one infers

$$
\int_{\tau}^{t}\left|\frac{\mathrm{d}}{\mathrm{d} t} \int_{\Omega} z^{2}\right| \leq C
$$

for $t \geq \tau$. Finally, the result follows by Lemma 3.3.

\section{Acknowledgments}

T. Cieślak was partially supported by the Polish Ministry of Science and Higher Education under grant number NN 201366937. C. Morales-Rodrigo was supported by Ministerio de Ciencia e Innovación and FEDER under grant MTM2009-12367. Both authors are grateful to anonymous referees for their careful reading of the manuscript and suggestions which lead to an improved version of the paper.

Open Access. This article is distributed under the terms of the Creative Commons Attribution License which permits any use, distribution, and reproduction in any medium, provided the original author(s) and the source are credited.

\section{References}

1. Amann, H.: Nonhomogeneous linear and quasilinear elliptic and parabolic boundary value problems. In: Schmeisser, H.J., Triebel, H. (eds.) Function Spaces, Differential Operators and Nonlinear Analysis, Teubner Texte zur Mathematik, vol. 133, pp. 9-126 (1993)

2. Amann, H., López-Gómez, J.: A priori bounds and multiple solutions for superlinear indefinite elliptic problems. J. Differ. Equ. 146, 336-374 (1998)

3. Anderson, A.R.A., Chaplain, M.A.J.: Continuous and discrete mathematical models of tumor-induced angiogenesis. Bull. Math. Biol. 60, 857-899 (1998)

4. Cano-Casanova, S., López-Gómez, J.: Properties of the principal eigenvalues of a general class of non-classical mixed boundary value problems. J. Differ. Equ. 178, 123-211 (2002)

5. Delgado, M., Gayte, I., Morales-Rodrigo, C., Suárez, A.: An angiogenesis model with nonlinear chemotactic response and flux at the tumor boundary. Nonlinear Anal. TMA 72, 330-347 (2010)

6. Delgado, M., Suárez, A.: Study of an elliptic system arising from angiogenesis with chemotaxis and flux at the boundary. J. Differ. Equ. 244, 3119-3150 (2008)

7. Fontelos, M., Friedman, A., Hu, B.: Mathematical analysis of a model for the initiation of angiogenesis. SIAM J. Math. Anal. 33, 1330-1355 (2002)

8. Henry, D.: Geometric theory of semilinear parabolic equations. Lecture Notes Math. vol. 840, Springer (1981)

9. Kettemann, A., Neuss-Radu, M.: Derivation and analysis of a system modeling the chemotactic movement of hematopoietic stem cells. J. Math. Biol. 56, 579-610 (2008)

10. Levine, H.A., Sleeman, B.D., Nilsen-Hamilton, N.: Mathematical modeling of the onset of capillary formation initiating angiogenesis. J. Math. Biol. 42, 195-238 (2001)

11. López-Gómez, J.: The maximum principle and the existence of principal eigenvalues for some linear weighted boundary value problems. J. Differ. Equ. 127, 263-294 (1996)

12. Mantzaris, N.V., Webb, S., Othmer, H.G.: Mathematical modeling of tumor-induced angiogenesis. J. Math. Biol. 49, 183217 (2004) 
13. Tartar, L.: An introduction to Sobolev spaces and interpolation spaces. Lecture Notes of the Unione Matematica Italiana, vol. 3, Springer (2007)

14. Umezu, K.: Nonlinear elliptic boundary value problems suggested by fermentation. Nonlinear Differ. Equ. Appl. 7, 143155 (2000)

Tomasz Cieślak

Institute of Mathematics, Polish Academy of Sciences

Śniadeckich 8

Warsaw

Poland

e-mail: cieslak@impan.pl; T.Cieslak@impan.pl

Cristian Morales-Rodrigo

Dpto. de Ecuaciones Diferenciales y Análisis Numérico

Fac. de Matemáticas

Univ. de Sevilla

Calle Tarfia s/n

41012

Sevilla

Spain

e-mail: cristianm@us.es

(Received: March 22, 2012; revised: October 18, 2012) 